\title{
Virfual Reality Evacuation Experiments on Way-Finding Systems for the Future Circular Collider
}

\author{
Silvia Arias, Jonathan Wahlqvist, Daniel Nilsson and Enrico \\ Ronchi (1), Department of Fire Safety Engineering, Lund University, P.O. \\ Box 118, 22100 Lund, Sweden
}

Saverio La Mendola and Oriol Rios, CERN, European Organization for Nuclear Research, 1211 Geneva 23, Switzerland

Received: 7 August 2018/Accepted: 25 April 2019

\begin{abstract}
Evacuation times can be shortened in fire scenarios if people choose appropriate routes. Way-finding systems can be used to aid this process, thus their effectiveness needs to be evaluated. In the present study, the way-finding evacuation systems of the Future Circular Collider (FCC) of the European Organization for Nuclear Research (CERN) are investigated as its evacuation design presents several challenges from the fire safety perspective. To perform a comparison of different evacuation design solutions, a set of Virtual Reality (VR) experiments involving a total of 111 participants was performed. VR was used because the FCC facility is not built yet, and it allows for high experimental control and cost-effectiveness for comparisons of way-finding systems. The VR experiments reproduced a hypothetical fire emergency in which participants' egress behaviour was investigated. Three scenarios were represented, each of them adopting different evacuation safety concepts and way-finding systems. Different installations were included in the scenarios, such as scenario (1) flashing lights, scenario (2) static or dynamic signage (i.e. active and dissuasive signage indicating the direction towards or away from the danger) and scenario (3) a robot placed on a monorail on the vault of the tunnel, able to localize people in the tunnel and provide way-guidance information. Results show that the combination of red flashing lights at the exits, dynamic signage and a robot equipped with green flashing lights yielded the highest compliance to the way-finding intent of the system $(92.6 \%$ compliance vs $62.9 \%$ and $77.5 \%$ for scenarios 1 and 2 respectively).
\end{abstract}

Keywords: FCC, Evacuation, Fire safety, Virtual Reality, Way-finding, CERN, Particle accelerators

\section{Introduction}

The European Organization for Nuclear Research (CERN) has been performing a feasibility study concerning the construction of the Future Circular Collider (FCC) on the border between France and Switzerland. The FCC would be a cir-

\footnotetext{
* Correspondence should be addressed to: Enrico Ronchi, E-mail: enrico.ronchi@brand.lth.se
} 
cular accelerator that would increase in a tenfold the current collision energy of the Large Hadron Collider (LHC) [1, 2]. The perimeter of the accelerator is expected to be between $80 \mathrm{~km}$ and $100 \mathrm{~km}$ long, excavated between 300 and $600 \mathrm{~m}$ underground [3]. This information concerning the geometric layout of the FCC makes it easier to understand that such a type of facility presents unique challenges from evacuation perspective [4]. In particular, the presence of very long and deep underground tunnels may lead to distances between emergency exits/protected areas that may exceed the distances adopted in standard $\mathrm{road} / \mathrm{rail}$ tunnels (e.g. distances in American and European codes [5, 6]).

During a tunnel fire emergency, it is of crucial importance that evacuees take the appropriate actions in a timely manner [7]. In particular, the key decision to be taken in long underground tunnel accelerators is the direction of evacuation in the section that are not directly exposed to the threat (e.g. fire and smoke). The adoption of the wrong direction may lead people towards the fire threat, having possible negative consequences on human health due to the presence of smoke, heat and radiation [8]. In this context, way-finding systems are a useful technical solution to increase evacuees' level of safety by guiding them towards appropriate routes and exits [9]. This is subsequently associated with a lower probability of exposure to hazardous conditions.

The fire safety and evacuation literature has proposed and investigated the usage of different way-finding installations and systems for road and rail tunnels, intended as any means able to provide information to the evacuees on the correct route to adopt [10]. Way-finding systems have been proposed for both smoke-filled [11-13] and smoke-free environments [14], but their use and effectiveness has been tested mostly in accordance to current road/rail tunnel regulations, which generally advise for maximum distances between emergency exits/protected areas in the order of $300 \mathrm{~m}$ to $500 \mathrm{~m}[5,6]$. General guidelines exist concerning signage in tunnel, e.g. the use of ISO signs and symbols to provide safety information [15]. Given their unique configurations, particle accelerator tunnels may present longer distances between emergency exits/protected areas. They also require a safety standard which may be higher than other tunnel infrastructures, as there are potential risks associated with hazards that are uncommon in transportation tunnels (e.g. oxygen deficiency hazard associated to helium release scenario, etc.). For this reason, a necessary step for the feasibility study of the FCC design is the identification and evaluation of a set of the most advanced way-finding installations available today. The selection of the way-finding systems to be considered was made in accordance with three criteria (1) systems currently used at CERN in existing tunnel accelerators (2), the feasibility of implementation of new evacuation installations in the suggested FCC tunnel design, (3) the potential effectiveness of new systems. This includes the use of flashing lights [14, 16] and dynamic signage (such as dissuasive [17] and active signage [18]). A further innovative wayfinding installation investigated here that could potentially be implemented in such type of facility is a robot on a monorail which includes an intelligent device to locate evacuees (e.g. such devices may be based on radiofrequency identification (RFID) sensors [19, 20] or thermal cameras [21]) and then provide way-guidance information. Those technologies may already be implemented in particle accelera- 
tor tunnel facilities for other scopes (e.g. identification of people location, maintenance [22], etc.) or infrastructures which requires a higher level of safety (e.g. mines [19]), thus their use for safe evacuation is feasible.

To address the issues associated with the fire safety design of the FCC, CERN has initiated a collaboration with a set of international institutions (particle physics accelerator facilities and academic institutions) to share knowledge and perform joint research on the fire safety design of FCC. Specifically, one part of the collaboration relates to the evacuation-related aspects of the fire safety design [23]. The aim of this work was the identification and evaluation of different evacuation design solutions for the FCC; these may include different fire safety-related technical installations.

This paper presents the results concerning possibly suitable evacuation design concepts which have been identified and compared for the deep and long sections of the accelerator tunnel. In particular, the main aim of this study is the investigation of the effectiveness of different systems aiming at providing way-finding information to evacuees in case of fire emergencies. The study has been performed with test participants which resemble the population that may be in an underground tunnel accelerator facility. The objectives of the work are therefore (1) the assessment of the suitability of a set of proposed evacuation design solutions including way-finding systems for the FCC accelerator tunnels, (2) the comparison of the performance of different evacuation design solutions and (3) the evaluation of the use of VR as a method for evacuation research of facilities that are not built yet, based on the realism of the scenarios perceived by the participants during the experiments.

\section{Evacuation Experiments of the FCC Tunnel}

As the FCC represents a unique facility which is currently under study, Virtual Reality (VR) experiments - a novel methodological approach in evacuation research $[24,25]$ - have been used to investigate different way-finding installations and the evacuation design concept of the FCC. Virtual Reality evacuation scenarios allow participants to experience a simulated emergency scenarios so that their behaviour can be observed.

Its use is proposed here as it presents several advantages for the evaluation of the effectiveness of different way-finding aid installations. In particular, VR allows people feeling present in a virtual environment [26], thus this experimental methodology is extremely suitable for unique facilities that are not built yet, as it would not be possible to investigate the behaviour of people in a simulated emergency scenarios in the actual facility. In addition, VR allows for high experimental control [27], thus giving the possibility to easily manipulate the environment and the evacuation installations present in the tunnel where the emergency scenario takes place.

Different VR technologies are available today that have been used in the context of tunnel evacuation research, including Cave Automatic Virtual Environments [16, 28], Head Mounted Displays (HMD) [9], mobile-phone powered head 
mounted displays [29], etc. The experiments presented here have been conducted with an Head Mounted Display technology (HTC Vive ${ }^{\mathrm{TM}}$, dual AMOLED 3.6" diagonal screen, $1080 \times 1200$ pixels per eye, $90 \mathrm{~Hz}$ refresh rate and 110 degrees of field of view, and 6 degrees of freedom of movement) as it gave the possibility to conduct experiments directly on the sites where existing accelerators are located. This facilitated the recruitment of a population relevant to the study and permitted to perform experiments at different locations (as Head Mounted Displays are easily transportable).

Prior to starting the recruitment and conducting the experiments, ethical and legal clearance to conduct the experiments were granted by CERN and ESS. Once participants volunteered to take part in the experiments, background information about the possible risks associated with the experiment (e.g. possible nausea or dizziness during the navigation in the virtual environment) were provided. It should be noted that the research team at Lund University had also obtained (before the experiments were conducted) a decision from the regional Swedish ethical board that evacuation experiments performed in Virtual Reality do not present significant risks to health and safety, thus they do not need to go through national ethical review before being conducted. Nevertheless, an internal ethical evaluation was performed prior to conducting experiments to assess possible risks and those were communicated to the participants prior conducting the experiments. This evaluation also excluded from the experiments people who had epilepsy (this was included in the recruitment information). Participants were taking part to the experiments as part of their work, thus no financial reimbursement was provided to them. Personal data were stored according to data protection routines at the Department of Fire Safety Engineering at Lund University. Data access and the virtual environment can be requested contacting the corresponding author of the manuscript.

The VR environment consisted of a portion of a particle accelerator tunnel based on one of the preliminary designs of the FCC. It was originally drawn using a 3D modelling software (SketchUp ${ }^{\mathrm{TM}}$ version 2017) and then imported into a game engine (Unity3 $\mathrm{D}^{\mathrm{TM}}$, version 5.6.3p2). The equipment represented in the virtual tunnel was based on those present in existing tunnel particle accelerators (e.g. the Large Hadron Collider at CERN, see Fig. 1 for a comparison of an actual tunnel accelerator and the FCC virtual environment).

\subsection{Participants}

A total of 111 participants took part in the experiment $(77$ male, 32 female and 2 preferred not to say), of whom 79 people were employees at CERN and 32 people were employees from other particle physics facilities (European Spallation Source, ESS [30], or Max IV laboratory [31]). Test participants' age ranged from 19 to 60 years old (average $=31.7$ years old and standard deviation 7.9 years). More than three quarter of the participants had no involvement in the health and safety field $(76 \%)$. The participants were well distributed in terms of their years of experience working in a tunnel accelerator facility, including 20 people who have worked there for less than a year, 70 people between 1 year and 5 years and 20 

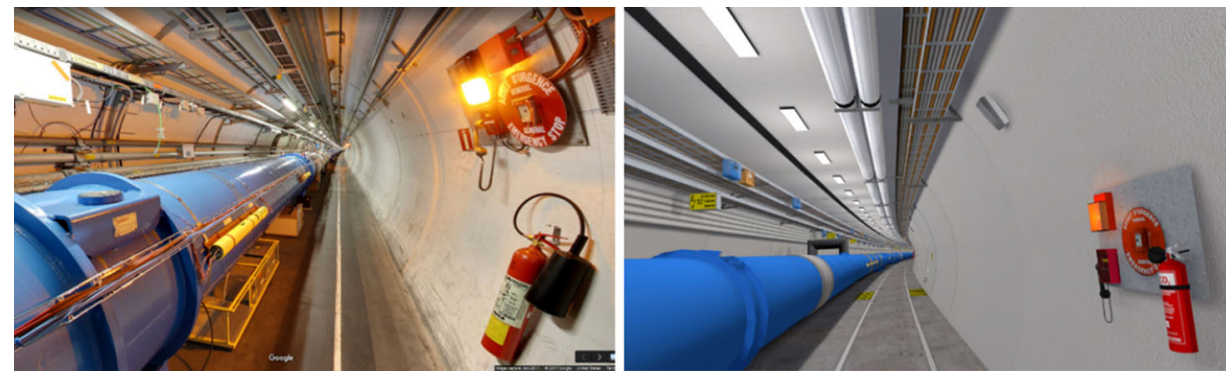

Figure 1. Actual tunnel accelerator configuration (of the Large Hadron Collider at CERN, taken from Google street view, retrieved in 2017 , with a cross section of approximately $3 \mathrm{~m}$ ) on the left versus virtual tunnel environment of the Future Circular Collider on the right, with a cross section of approximately $6 \mathbf{m}$.

people for more than 5 years (1 person preferred not to say). All participants recruited at CERN were employees there, while among people recruited at ESS, 21 people never worked at CERN while the rest had done so. Over half of the participants had been in an accelerator tunnel (59 people) at least once. It should be noted that people working at particle accelerators are required to perform a mandatory safety training before accessing the underground facility. The recruitment phase therefore attempted to match the population which could potentially be present in tunnel accelerators, i.e. both people that are familiar with tunnel accelerators as well as people with less familiarity (this is given the fact that this type of facilities may host contractors or visitors which may be underground for a relatively short time).

Participants were recruited via email through the mailing lists available at CERN and at the ESS. The content of the email for recruitment was written in a way to not disclose the scope of the experiment, i.e. participants knew they were going to try a virtual environment representing the FCC but they were not aware that they were going to be exposed to an emergency evacuation experiment. This was intended to not affect the behaviour of people expecting an emergency from the beginning. Participants were then given a schedule with possible times in which they could sign up for the experiment. Once they chose a free time slot, they were given a confirmation on the time and place where the experiment would have taken place.

\subsection{The Environment}

The virtual environment consisted of a segment of a tunnel accelerator equipped with a set of way-finding systems. The tunnel environment and these systems are here described.

2.2.1. The Virtual Tunnel The virtual tunnel environment consisted of a segment of $440 \mathrm{~m}$ length with a cross section of $6 \mathrm{~m}$ in diameter according to a possible design configuration of the FCC layout. Slightly less than half the cross section is 
available as walkable area (the other half is occupied by the particle accelerator). These dimensions were selected as they represented a proposed size of the compartment at the time the experiments were conducted. The virtual tunnel was given a curvature different than the one of the FCC so that the doors at the end of the tunnel compartment could not be visible from the initial position of the participants. Exit doors to the other compartments were located at the ends of the compartment.

Procedural generation [32] was the selected method to create the VR tunnel segment. This method generates the environment algorithmically, and therefore allows minimizing the time invested in generating the geometry and at the same time adds variation along the environment. A $5 \mathrm{~m}$ long tunnel section was drawn as a base, consisting on the walls, ceiling, floor, lighting, piping and cable installations. This base element would be placed along the entire segment. Additionally the different components of the magnet and details such as safety equipment and emergency signalling were drawn individually. The procedural generation script developed for this scenario was able to take each of those components and place them along the length of the $440 \mathrm{~m}$ segment. In the case of the accelerator magnet [33], the components were placed randomly since it was not necessary to present an exact replica of the real magnet. In addition, some extra details, like electrical boxes, floor hatches, warning signs (e.g. radiation, electrical hazard and helium release) were placed randomly to give variation along the tunnel. The safety equipment and emergency signalling were placed at given distances, since this is the way that such kinds of installations are placed in reality. The procedural generation script also allowed selecting a frequency factor for each component as some components are more likely to appear than others. At each end of the segment a compartment wall with a double door was placed (see Fig. 2). The script was written in such way that the length and the curvature of the segment to be generated could easily be changed. This allows reuse of the scenario and adapt it to any length needed.

When a tunnel segment had been generated, it was visually inspected to check if the procedurally added variation gave a credible output. Once the output was deemed satisfactory, the generated tunnel was saved and used for the compiled version of the scenario. This ensured that every participant was exposed to the exact same configuration in each scenario.

The evacuation path was assumed free of obstacles. This is the most likely scenario as even in case there are obstacles in a real tunnel accelerator, those would not completely impede passage towards one direction. There are actually no large obstacles introduced in the tunnel other than those in transit.

The experimental scenarios and procedures were first analysed with pilot testing in order to make sure that the scenario was working as intended.

2.2.2. Way-Finding Systems The selection of the way-finding systems tested in the virtual accelerator tunnel included different combinations of installations or systems. A preliminary literature review was conducted by the project team at CERN and Lund University to investigate existing and novel way-finding aid systems in case of evacuation in line with the specific evacuation-related issues of particle 

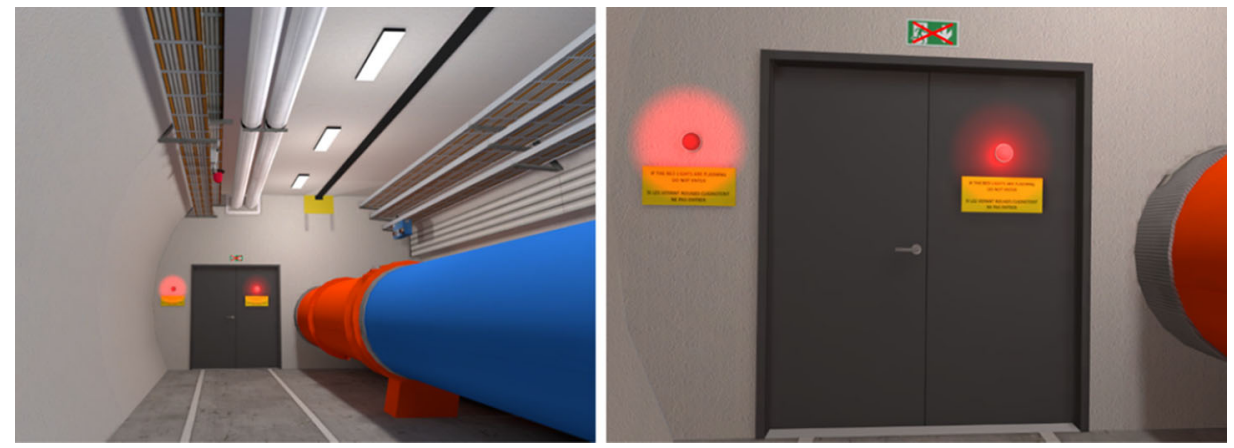

\section{Figure 2. Configuration of the door and signage at the end of the tunnel compartment. The red flashing lights and the written message with the yellow background are shown. The cross section of the funnel is approximately 6 m.}

accelerator tunnels [4]. This included lighting and acoustic systems [9, 14, 16, 3439], existing installations available in tunnel accelerators that could get an additional evacuation aid function (i.e. the Train Inspection Monorail (TIM) robot) [22] and evacuation signage [17, 18, 40-42].

The chosen setups were the result of this literature review and the design solutions selected for testing were deemed to be way-finding aid solutions with the highest effectiveness.

The final selected way-finding systems design has been chosen since it reflects the current design adopted in the Super Proton Synchrotron (SPS) facility at CERN [43]. This included a dissuasive system at the door of the tunnel compartment behind the threat including flashing lights at $1 \mathrm{~Hz}$ (this frequency was chosen based on its effectiveness for evacuation aid [16]) and a yellow sign with the following text "If the red lights are flashing do not enter" written in both English and French as these are the official languages at CERN. The Exit sign on top of the door also had a cross on its top to indicate that such door should not be used (see Fig. 2).

An additional way-finding system suggested for the accelerator tunnel consisted of the Train Inspection Monorail robot. This system has been chosen given its possible presence in tunnel accelerator facilities for reasons other than evacuation aid, as well as after assessing the feasibility of its implementation. This robot can be equipped with a set of way-finding installations which include (See Fig. 3):

- An emergency exit sign with the running man symbol [15] on an LED screen pointing the direction of evacuation

- A projection on the floor which indicated the direction of movement opposite to the robot with arrows and written text "Exit" in green.

- An acoustic sound (e.g. a mechanical bell).

An "improved" version of the Train Inspection Monorail robot included also an additional green flashing light at $1 \mathrm{~Hz}$ on the bottom of the robot. 

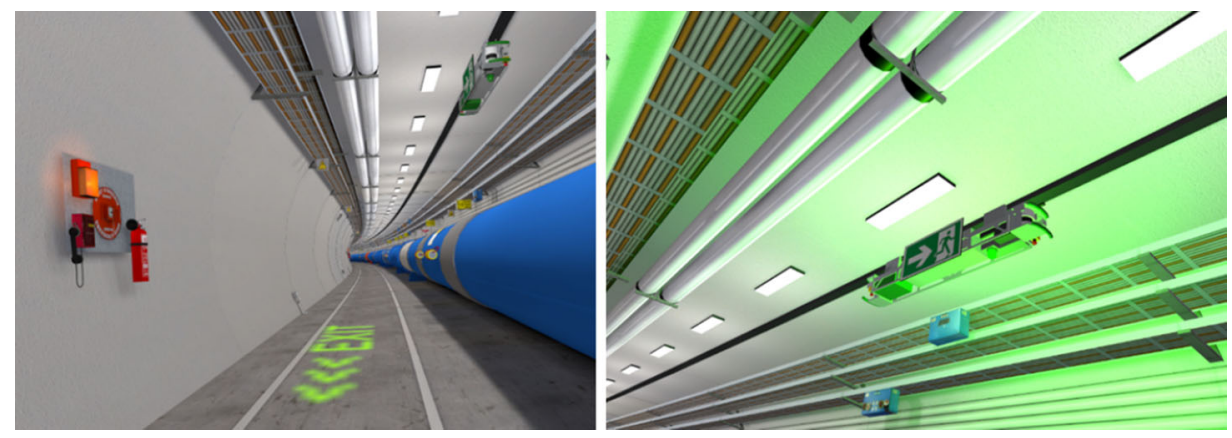

Figure 3. Configuration of the way-finding installations on the Train
Inspection Monorail robot in the virtual reality scenario. The
figure shows the projected "'Exit" message on the floor along with
the arrow indicating the recommended direction of movement.

The final suggested way-finding system to be tested consisted of a set of dynamic way-finding signage systems (placed every $55 \mathrm{~m}$ on the tunnel walls, this distance is based on a possible design of the FCC tunnel), which includes active and dissuasive signage indicating the correct direction of movement (See Fig. 4). The design of this way-finding installation has been based on previous research studies on active signage $[17,40]$.

The sound was reproduced in VR in $3 \mathrm{D}$. The actual sources of the sound were matching the location of the alarms in the planned tunnel design. This includes the sound from the robot which was generated from the robot itself. The sound of the alarms came from speakers located every $20 \mathrm{~m}$.

\subsection{Procedure}

The experiments took place in two sessions, one at CERN in Geneva (Switzerland) in November 2017 and one at ESS in Lund (Sweden) in February 2018. Each participant took part in the experiment one at a time. This choice was made to isolate individual behaviour rather than investigating social influence (of one more individuals) and it is in line with what is typically made to test designs [44, 45]. Upon arrival, he/she was welcomed by a researcher and provided with general written information about the experiment, which included both safety measures and informed consent. The informed consent included information concerning safety and privacy, including confidential treatment of personal data. In addition to written information, participants were also given oral information prior conducting the experiments. A number of precautions were taken in order to minimize the risk of physical injuries during the experiment. These included the presence of a researcher during the whole duration of the experiments and the possibility to seat in case of nausea/dizziness.

After getting the informed consent signed, participants were then guided to the location where the Head Mounted Display was placed (see Fig. 5). During the experiments, one researcher was in the proximity to help the participant in wear- 


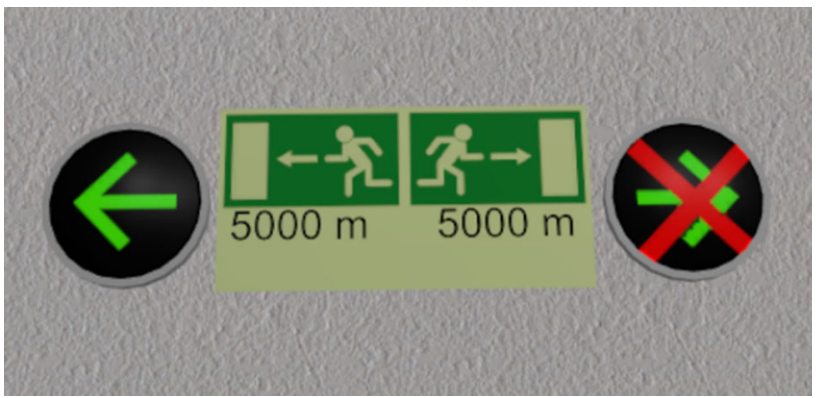

Figure 4. Example of configuration of the dynamic signage system on the funnel walls in the virtual reality scenario. The right sign has a red cross on it to indicate that this is the wrong direction of movement.

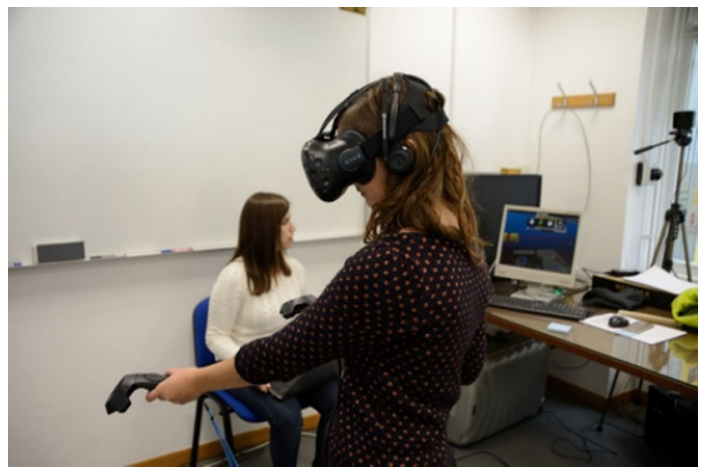

Figure 5. Participants taking part in the VR experiment. The participant wears the Head Mounted Display and the researcher can follow her behaviour in real time on a monitor.

ing the Head Mounted Display equipment and run the experiment (see Fig. 5). Participants were requested to wear the Head Mounted Display and to take the VR controllers in their hands. Participants then received a brief introduction about the equipment, i.e. how to operate the controllers to interact with objects in the VR world and how to navigate/move in the virtual environment. In fact, participants used the HTC Vive tracking controllers to navigate and interact with the virtual environment. Prior to starting the actual experiment, participants were one more time reminded that they could quit the experiment at any time if they were feeling dizziness/nausea or for any other reason. After participants declared to have understood the mechanisms for navigation and interaction with the virtual space, the actual experiment started. Participants then received VR training both as instructions and as a task in VR at the beginning of the scenario to get familiarized with the equipment. 
Participants were initially located in the centre of the $440 \mathrm{~m}$ virtual accelerator tunnel segment and they were given a bogus task in order to test the interaction with the environment. The bogus task consisted in grabbing a set of objects/tools present in the virtual environment and place them inside of a box. Participants did not know that at a specific point of the bogus task the fire alarm was going to be triggered. The alarm consisted of a siren with a pitch of varying frequency, currently used in the Large Hadron Collider tunnel. The environment was smokefree as it was assumed that the fire was in a different compartment than that of the participant, and the compartments are assumed to be smoke-tight. This assumption was made as it represents the worst case scenario in evacuation (people do not have direct cues of the threat and they may need to take a decision on the direction to take without knowing the location of the threat). Given the size of the compartment, the presence of smoke would be linked to visual and olfactory cues before reaching reduced visibility. In this case it is expected that the occupants would be more likely able to tell from which direction the threat is coming. The uncertainty about the type of threat could be achieved only without having participants in the compartment of the threat. In addition, this allowed the scenario to be general enough to possibly cover other possible evacuation emergencies than fire (e.g. radiological, cryogenic release, etc.).

The main behaviour observed was the evacuation direction taken by the participants in relation to the way-finding information received. In fact, regardless of the chosen direction of movement, the virtual scenario was designed to signal the participants that they should evacuate in the opposite direction. Therefore, participants would either move until they reach a door of the compartment and be exposed to the red flashing lights and signage providing information on not using that door, or be reached by the robot on the rail which would provide instructions to go towards the opposite direction of movement. In both cases, participants could decide to follow the instructions received and change direction of movement or ignore it. Once they made their decision (i.e. to enter the door to the other tunnel compartment or change direction) and their compliance to the instructions received was recorded, the experiment stopped. During the experiment, the researcher was able to see what participants were seeing at all times, and a video of each participants' behaviour in the virtual tunnel was recorded.

After completion of the VR experiments, the participants were helped by the researcher to remove the Head Mounted Display and the VR controllers and they were given a questionnaire to fill in which included both background questions (age, gender, involvement with health and safety, years of experience working at CERN and experience with tunnel accelerator facilities) as well as questions concerning the VR experience. The latter included questions concerning the actions and decisions taken by the participants as well as the level of perceived realism of the scenario. The experience of each participant during the VR scenario was also investigated by asking them to rate their levels of insecurity, stress, fear, disorientation, and physical discomfort. 


\section{Table 1}

Installations in the Tunnel Evacuation Scenarios

\section{Scenario num-}

ber Way-finding systems

Scenario $1 \quad$ Baseline with flashing lights and signage on the tunnel door

Scenario 2 Flashing lights and signage on the tunnel door + TIM robot

Scenario 3 Flashing lights and signage on the tunnel door + Improved TIM robot + dynamic signage

Three scenarios have been represented including a combination of different way-finding systems

\subsection{Tunnel Evacuation Scenarios}

The evacuation experiments were conducted considering three scenarios which included a combination of different way-finding installations. The installations present in each scenario are presented in Table 1. Scenario 1 is the baseline scenario, which includes a configuration of evacuation installations inspired on some of the existing CERN facilities. An iterative process of scenario definition was employed for the definition of scenarios 2 and 3. First the Train Inspection Monorail robot was added in Scenario 2 and then an improved version of the robot with an additional green flashing light as well as dynamic signage was used in scenario 3 in order to further improve the design. Scenario 2 and 3 represent then improved evacuation designs which were then compared to the baseline to evaluate if they increase the compliance to the way-finding instructions provided.

Participants were randomly assigned to one of the scenarios (with the exception of participants taking part of the second session of experiments at ESS who were assigned to the third scenario, as this was designed later as an improvement of scenario 2 and they started navigating in the tunnel and receiving way-finding aid from the evacuation installations adopted in the given scenario. It should be noted that the experimental procedure and the hardware configuration were kept the same between the experiments at CERN and ESS.

\section{Results}

Questionnaire responses were collected in order to have participants evaluating the VR experience and the realism of the virtual environment. The main behaviour observed in the VR environment related to the percentage of people who were compliant to the instructions provided by the way-finding systems in relation to the scenarios they were experiencing. This allowed a comparison between different evacuation designs as the scenarios included different way-finding installations. Participants were also given the opportunity to provide qualitative open comments on the evacuation scenarios and the virtual environment. 


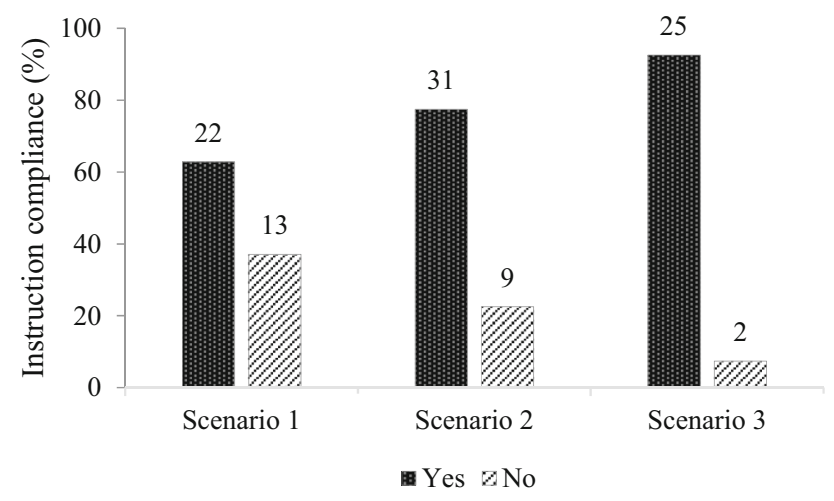

\section{Figure 6. Summary of results concerning instruction compliance.}

\subsection{Percentage of Instruction Compliance}

Figure 6 presents the people who took the decision to modify their direction of movement in relation to the way-finding information provided by the evacuation installations system present in the scenarios. This could be the result of the signage system on the door (scenario 1), signage system on the door and the Train Inspection Monorail robot (scenario 2) or signage system on the door, the improved robot and the dynamic signage (scenario 3). It should be noted that the decisions of some participants could not be collected while two participants changed their direction of movement prior interacting with the way-finding systems (for these reasons, these data have been removed from the results). Those participants could not explain the reasoning for changing direction.

Fisher exact testing (significance level with Bonferroni correction equal to 0.025) was performed to compare the Baseline scenario with the scenarios having additional evacuation installations. Results show that the results of scenarios 1 and 2 are not significantly different, while the differences in results between scenarios 1 and 3 are statistically significant (Fisher exact test statistic value is $0.0076<0.025$ ). Therefore, this indicates that the combination of evacuation installations in scenario 3 (flashing lights and signage on the door plus improved Train Inspection Monorail robot plus dynamic signage) increase the instruction compliance compared to the baseline scenario.

\subsection{Questionnaire Results}

The questionnaire performed after the experiments included a set of questions which relate to the participants' experience of the VR environment. Participants were asked to rank from 1 to 7 (from none to high) the following six sensations experienced during the vr tests, namely (1) insecurity, (2) stress, (3) fear, (4) disorientation, (5) physical discomfort (dizziness and nausea), (6) Physical discomfort (eye problems). These questions were made both to address ethical considerations (i.e. to assess whether the VR experiments are associated to extreme values) as well as investigate the level of perceived urgency. To avoid misinterpretation of 

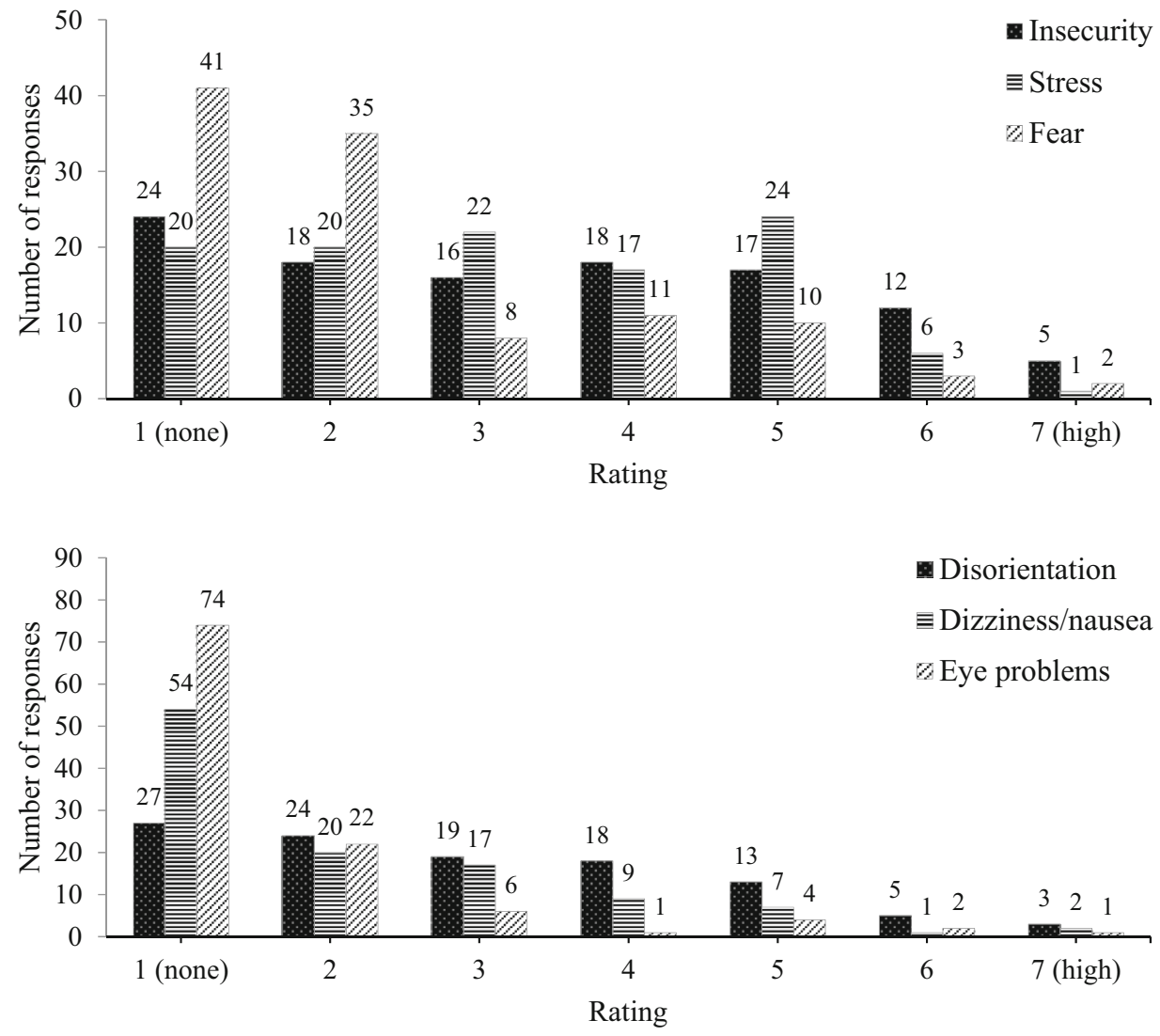

Figure 7. Number of participants' responses to the questions concerning the VR experience. This includes insecurity, stress, fear, disorientation, dizziness/nausea and eye problems. Rating from 1 (none) to 7 (high).

the responses by assuming linearity in the scale, the responses are shown as histograms. The participants were asked to rate the sensations from 1 (none) to 7 (high). The responses of the participants are presented in Fig. 7 (110 responses out of 111 participants were collected for all these questions with the exception of the question on disorientation in which 109 responses were collected). The figure shows the number of participants giving the same rating to each sensation.

Results on VR experience are presented in Table 2. They seem to indicate a reduced level of discomfort given by VR experiments.

Non-parametric testing (Kolmogorov-Smirnov) was also performed to compare the pairs of six scores concerning the VR experience between the participants that took part to the experiments at CERN and the ones who took part at ESS. No statistically significant differences were found (insecurity $\mathrm{D}=0.0846, P=0.997$; stress $\mathrm{D}=0.1077, \quad P=0.952 ;$ fear $\mathrm{D}=0.1000, \quad P=0.975$; disorientation 


\section{Table 2}

Summary of Descriptive Statistics Concerning Participants" VR Experience

\begin{tabular}{lcccccc}
\hline & Insecurity & Stress & Fear & Disorientation & Dizziness/nausea & Eye problems \\
\hline Mean & 3.4 & 3.2 & 2.4 & 2.9 & 2.2 & 1.6 \\
Standard deviation & 1.8 & 1.6 & 1.5 & 1.7 & 1.5 & 1.2 \\
\hline
\end{tabular}

$\mathrm{D}=0.1801, P=0.446$; dizziness $/$ nausea $\mathrm{D}=0.2538, P=0.103$; eye problems $\mathrm{D}=0.1846, \quad P=0.413$ ), thus corroborating the hypothesis that the results obtained are not dependent on the location where the experiments were performed.

A set of questions were also made to participants in order to investigate their level of perceived realism and included (1) the sound alarm, (2) the tunnel end (including flashing lights), (3) the robot, (4) emergency signage (exit signs, distance signs, emergency points), (5) the whole tunnel environment, (6) the accelerator. Such questions may depend on the scenario which the participants experienced (as evacuation installations were different). Participants were asked to rate the realism of those elements from 1 (low) to 7 (high). Figure 8 shows the number of participants giving the same rating for each element.

It can be noticed that the scores concerning the realism of the perceived environment were giving average scores of 5.8 (sound alarm), 5.5 (tunnel end), 5.4 (robot), 5.7 (emergency signage), 5.5 (the whole tunnel environment) and 5.4 (accelerator). This seems to indicate that participants considered the VR environment fairly realistic.

An additional question was also made to investigate the level of presence during the VR experiments, i.e. people were asked to which extent people forgot that they were taking part into a simulated evacuation emergency. The majority of people (51 responses) declared to have forgotten to be in a virtual environment, 34 people declared that they did not forget and 24 declared that forgot it to some extent ( 2 people did not respond).

\subsection{Qualitative Comments}

Participants were given the possibility to comment on their actions and the decisions that they took during the VR experiment as well as the VR evacuation experience itself. One question asked participants to describe the actions they did once they heard the alarm going off. The majority of participants mentioned that they were looking around in order to get information about the situation and identify the source of the alarm. When asked about the cause of the sound alarm, the most frequent response (26 participants) was that they did not know the cause of the alarm, while others mentioned that they understood that an evacuation was required. People interpreted the sound alarm with different type of emergencies, 

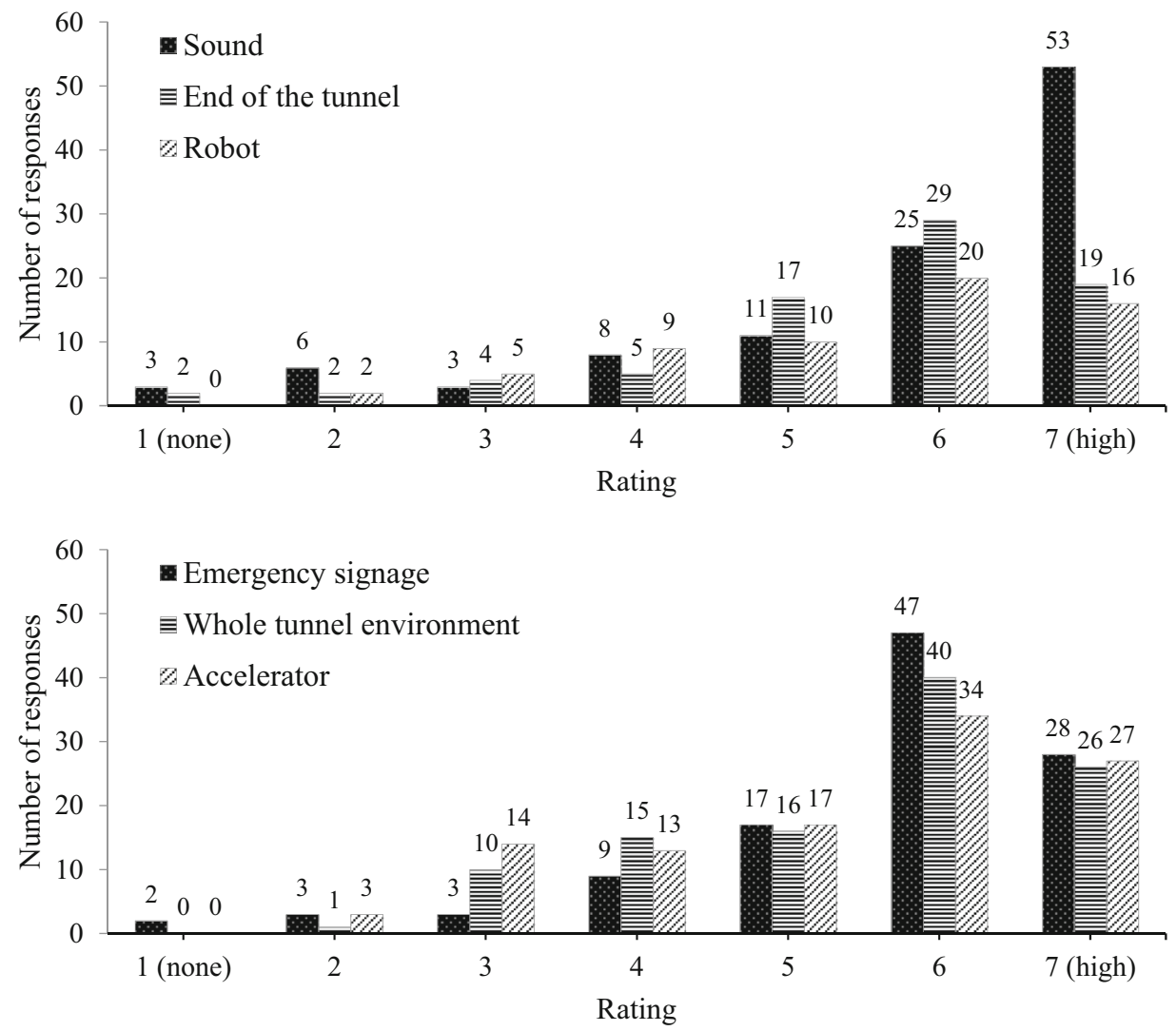

Figure 8. Number of participants' responses to the questions concerning the perceived realism of the VR environment. This includes realism of sound, end of the funnel, robot, emergency signage, whole tunnel environment and particle accelerator. Rating from 1 (low) to 7 (high).

such as a helium release, a fire, a radiation issue or the start of the accelerator beam.

Participants were also asked how they chose the direction of movement. Participants mentioned to have received different type of information to identify the correct direction of movement. Some participants clearly received the message from a single evacuation installation, for instance one participant mentioned:

I saw the sign that was moving and followed that one,

while another mentioned:

following the exit letters wrote on the floor

(they both refer to the sign projected by the robot). Some participants explicitly mentioned the dynamic signage: 
I saw the sign on the left wall with a red cross over and walked back in the opposite direction.

Other participants referred to the combination of information and how they reinforced the instructions given:

I looked around and saw several indications for the closest emergency exit. One of them was moving on a rail, the other was fixed on the wallor

There was a robot on the rails indicating to go for the exit and then also a visual sign on the floor, both telling you in which direction to go.

\section{Discussion}

The VR experiments presented in this paper have given the possibility to compare and evaluate a set of way-finding systems for the FCC evacuation design. The designs provided a compliance to the instructions given ranging from $62.9 \%$ to $92.6 \%$. The solution tested in scenario 3 (the combination of flashing lights and signage on the door plus the improved Train Inspection Monorail robot and the active signage) provided a compliance level which is significantly greater than the baseline design (i.e. flashing lights without the robot). Results suggest that the design including a combination of multiple evacuation installations and including active signage can significantly improve way-finding.

The use of Virtual Reality as a research method allowed the obtainment of a substantial amount of data since 102 individual way-finding behaviour were collected and 1224 responses (12 per participant) concerning the VR experience and perceived realism were obtained with a relatively good cost effectiveness. It should also be noted that since the FCC facility is a unique facility that has not been built yet, VR can be considered an ecologically valid methodology to collect data on human behaviour in evacuation emergencies [46]. While evacuation drills in actual tunnel accelerators would be valuable, such type of drills would not be possible as a configuration such as the one proposed for FCC today does not exist. In addition, it is very difficult to arrange drills in existing particle physics tunnels (e.g. the Large Hadron Collider at CERN) given their lack of accelerator down time.

Results should also be analysed in light of the fact that the Head Mounted Display adopted for the VR experiments has a field of view which is different than the one available to people in the real world. The natural field of view of a human is approximately 180 degrees, while the HTC Vive offers a 110 degree field of view. Thus the behaviour observed in such type of VR experiments which include the interaction with evacuation installations initially located outside the field of view (e.g. the Train Inspection Monorail robot) should be considered as a conservative approximation of the real world behaviour, i.e. people in real life might notice evacuation installations even more. 
Results were collected recruiting participants at different tunnel accelerator facilities. It should be noted though that a portion of the participants taking part to the experiments at ESS were previously employed at CERN or had been working with accelerator tunnels (see section concerning participants), thus the two populations at CERN and ESS can be assumed comparable. For this reason, the assumption that the use of two locations for running the scenarios is not influencing the results has been made (this has also been tested statistically, see Sect. 3.2).

The ecological validity of virtual reality evacuation experiments can be linked to previous studies comparing human behaviour in VR and in reality [46, 47].. The results of these experiments are in line with previous experimental research conducted in the real world aiming at investigating some of the evacuation installations systems under consideration, e.g. active signage and flashing lights [14, 42]. In addition, as the evacuation installation systems mostly consisted in visual and acoustic systems, the VR environment represented a valid alternative to physical experiments (as results concerning physical interactions in VR would inevitably be less ecologically valid [24]). Given the great flexibility and repeatability of VR - i.e. allowing in a very simple manner to add evacuation installations in the virtual environment - such experimental methodology has been a valuable tool to address the research questions under consideration in this study.

The behaviour observed as well as the qualitative comments made by participants seem to suggest that the combination of multiple evacuation installations can increase instruction compliance, i.e. the likelihood that people would follow an instruction about their direction of movement tends to increase if such information is given by multiple systems. This finding is in line with existing human behaviour in emergency theories which state that people tend to take their decisions on the basis of multiple cues [48, 49]. In addition, the uncertainty associated with the identification of the meaning of the type of alarm should make safety designers reflect on the need to adopt a single evacuation alarm sound rather than multiple sounds associated with different threats (e.g. fire, radiological, etc.).

From a technological standpoint, the VR technology in use (an Head Mounted Display) has not given a high level of physical discomfort to participants and not a single participant quit the experiment because of it. Along with the fact that people are not exposed to a real emergency, this makes VR a valuable methodological tool for evacuation research from an ethical point of view. Future research may aim at improving the levels of insecurity, stress, fear and disorientation (and in general the level of perceived urgency) by adding additional stimuli to the scenario (such as olfactory or haptic feedback [50]) in order to further improve the validity of the results. Nevertheless, the trade-offs between ethical restrictions and realism of the experience should always be taken into consideration while conducting such type of evacuation emergency research studies [51]. It should also be noted that some participants who did not exhibit instruction compliance declared that were interested in testing the limits of VR, in particular considering the participants adopting a "gamer" approach during VR experiments. This limitation should be taken into consideration while attempting to use VR tools for training, e.g. serious games for evacuation training [52] and while analys- 
ing the results. Despite this issue, given the observed compliance to the way-finding instructions provided, results are deemed to be applicable for the FCC design.

Some considerations need to be made on the generalizability of results given the impact of the sample under consideration in the present evacuation experiments. Participants were recruited among people working in accelerator tunnel facilities (CERN, ESS and MaxIV) in an attempt to match the possible type of population present in such type of facilities. Given the scope of the study (the evaluation of the evacuation design for the FCC), such sample is deemed to be relevant and results applicable for the FCC. Nevertheless, care should be made in generalizing the results outside this population type as general public who is not familiar with particle physics facilities might have a different response to installations that are not familiar to them (e.g. the Train Inspection Monorail robot). A careful evaluation of the findings is therefore needed in order to generalize the results of this paper for other type of infrastructure facilities (e.g. road and rail tunnels) given possible differences in familiarity with the facility (as the population in road/rail is overall less familiar with the environment) as well as architectural features (e.g. differences in cross sections and typical use of the facility).

It is believed that future studies will investigate the links between the perceived realism and the behaviour in VR evacuation (this should be ideally performed investigating several VR experiments with different level of sophistications and resolution in the scenario representation). Future research should focus on investigating an increased number of evacuation scenarios and evacuation installations. For instance, this can include the study of different evacuation installations in isolation or the investigation of the impact of different combinations of installations using larger sample sizes. In fact, the current study includes an uneven number of participants for scenarios 1 and 2 as we had to discard four participants in one scenario because they did not interact with the way-finding systems at all, while the third scenario presented a lower number of participants due to recruitment issues (given the specific type of participants needed). In addition, VR experiments have been conducted with people navigating the virtual environment one at a time, while social influence can have a significant impact on evacuation performance, as demonstrated by previous VR experiments in tunnel evacuation scenarios [28]. Future studies should address how instruction compliance is affected by social influence, i.e. decision making in relation to the decisions of others [28, 44]. As the occupant load inside a tunnel accelerator is relatively low and the worst case scenario is actually the presence of a single individual in a tunnel accelerator (as previously investigated in [44]), the scenarios under consideration are still deemed to be relevant for the design of FCC. Future research should further investigate the interaction between evacuation installations and decision making in groups.

\section{Conclusion}

VR has been a powerful tool to compare evacuation installation effectiveness and investigate emergency scenarios in a unique facility that has not been built yet, the Future Circular Collider. The scenarios including different evacuation design con- 
cepts and way-finding systems were compared. Such scenarios represented the most suitable options for evacuation design in the FCC, thus having a direct applicability in the real world. Moreover, VR allowed testing different possible way-finding systems for this facility and indicated the most suitable design among the selected ones prior to implementation in the real world. As the results fall in line with previous real-world research on way-finding systems, the recommendations drawn from this study can be used for fire evacuation design. Results show that the combination of flashing lights, dynamic signage and a robot installed on a monorail provided a significantly better way-finding instruction compliance than a baseline scenario in which no dynamic signage and robot were installed.

\section{Acknowledgements}

This work is part of the collaboration framework between Lund University and the European Organization for Nuclear Research (CERN), concerning the feasibility study of the Future Circular Collider (FCC) (Addendum FCC-GOV-CC0052 (KE3193/HSE)). The purpose of this collaboration is to enhance the exchange of information for fire protection at physics research facilities. The members of the collaboration belong to particle physics research laboratories (i.e. CERN, ESS, Max IV, Fermilab and Desy) as well as academic institutions. The work presented in this report is a sub-part of "Work Package 4: Evacuation" of the collaboration. The authors wish to acknowledge Fredrik Jörud and Marina Giampietro (both at ESS), Eric Gard (Lund University), Mario Di Castro, Art Arnalich and Tristan Hehnen (CERN) for providing help and support in the organization of the VR experiments.

\section{Open Access}

This article is distributed under the terms of the Creative Commons Attribution 4.0 International License (http://creativecommons.org/licenses/by/4.0/), which permits unrestricted use, distribution, and reproduction in any medium, provided you give appropriate credit to the original author(s) and the source, provide a link to the Creative Commons license, and indicate if changes were made.

\section{References}

1. Benedikt M, Zimmermann F (2016) Towards future circular colliders. In: 19th international conference accelerators and beam utilizations ICABU2015 Gyeongju Repub. Korea Nov 4-6 2015, vol 69, no 6, pp 893-902

2. Benedikt M, Zimmermann F (2016) Future circular colliders. In: Proceedings, 27th international symposium on lepton photon interactions. High Energy LP15 Ljubl. Slov. August 17-22, 2015, vol LeptonPhoton2015, p 052

3. Benedikt M, Zimmermann F (2018) Proton colliders at the energy frontier. Nuc Instrum Methods Phys Res A 907:200-208 
4. Ronchi E, La Mendola S (2016) Evacuation modelling for underground physics research facilities. Department of Fire Safety Engineering, Lund University, Lund

5. NFPA (2017) Standard for road, tunnels, bridges, and other limited access highways (NFPA 502)

6. European Commission (2004) Directive 2004/54/CE on minimum safety requirements for tunnels in the Trans-European Road Network (29/04/2004)

7. Kinateder M et al (2013) Human behaviour in severe tunnel accidents: effects of information and behavioural training. Transp Res Part F Traffic Psychol Behav 17:20-32

8. Purser DA (2008) Assessment of hazards to occupants from smoke, toxic gases and heat. In: SFPE handbook of fire protection engineering, 4th edn. Di Nenno PJ, Quincy, pp 2-96-2-193

9. Cosma G, Ronchi E, Nilsson D (2016) Way-finding lighting systems for rail tunnel evacuation: a virtual reality experiment with Oculus Rift $^{\circledR}$. J Transp Saf Secur 8(sup1):101-117

10. Nilsson D (2009) Exit choice in fire emergencies: influencing choice of exit with flashing lights. Department of Fire Safety Engineering and Systems Safety, Lund University, Lund

11. Ronchi E, Fridolf K, Frantzich H, Nilsson D, Walter AL, Modig H (2017) A tunnel evacuation experiment on movement speed and exit choice in smoke. Fire Saf J 97:126136

12. Boer LC, van Wijngaarden SJ (2004) Directional sound evacuation from smoke-filled tunnels. Innovative European Achievements, Prague, Czech Republic, Presented at the safe and reliable tunnels

13. Heskestad AW (1999) Performance in smoke of wayguidance systems. Fire Mater 23(6):375-381

14. Nilsson D, Frantzich H, Saunders W (2005) Coloured flashing lights to mark emergency exits - experiences from evacuation experiments. Fire Saf Sci 8:569-579

15. International Standards Organization (2011) ISO 3864-1:2011 Graphical symbols - safety colours and safety signs

16. Ronchi E et al (2016) A virtual reality experiment on flashing lights at emergency exit portals for road tunnel evacuation. Fire Technol 52(3):623-647

17. Olander J, Ronchi E, Lovreglio R, Nilsson D (2017) Dissuasive exit signage for building fire evacuation. Appl Ergon 59:84-93

18. Galea E, Xie H, Lawrence P (2014) Experimental and survey studies on the effectiveness of dynamic signage systems. Fire Saf Sci 11:1129-1143

19. Bhat AS, Raghavendra B, Kumar GN (2013) Enhanced passive RFID based disaster management for coal miners. Int J Future Comput Commun 2(5):476

20. Bolic M, Simplot-Ryl D, Stojmenovic I (2010) RFID systems: research trends and challenges. Wiley, New York

21. Fridolf K, Ronchi E, Nilsson D, Frantzich H (2013) Movement speed and exit choice in smoke-filled rail tunnels. Fire Saf J 59:8-21

22. Di Castro M, Masi A, Gilardoni S, Losito R, Baiguera Tambutti ML, Lunghi G (2018) JACoW: LHC train control system for autonomous inspections and measurements

23. La Mendola S, van Hees P (2016) Memorandum of understanding ADDENDUM FCC-GOV CC-0052. CERN

24. Kinateder $M$ et al (2014) Virtual reality for fire evacuation research. In: 1st workshop "complex events and information modelling," Warsaw, pp 319-327

25. Bode NWF, Miller J, O'Gorman R, Codling EA (2015) Increased costs reduce reciprocal helping behaviour of humans in a virtual evacuation experiment. Sci Rep 5(1):15896 
26. Steuer J (1992) Defining virtual reality: dimensions determining telepresence. J Commun 42(4):73-93

27. Duarte E, Rebelo F, Wogalter MS (2010) Virtual reality and its potential for evaluating warning compliance. Hum Factors Ergon Manuf Serv Ind 20(6):526-537

28. Kinateder $M$ et al (2014) Social influence on route choice in a virtual reality tunnel fire. Transp Res Part F Traffic Psychol Behav 26:116-125

29. Ronchi E, Mayorga D, Lovreglio R, Wahlqvist J, Nilsson D (2019) Mobile-powered head-mounted displays versus cave automatic virtual environment experiments for evacuation research. Comput Animat Virt W . https://doi.org/10.1002/cav.1873

30. Peggs, S. et al. (2012) ESS Conceptual Design Report. Research Report. European Spallation Source, Lund, Sweden

31. Tavares PF, Leemann SC, Sjöström M, Andersson $\AA$ (2014) The MAX IV storage ring project. J Synchrotron Radiat 21(5):862-877

32. Smelik R, Tutenel T, de Kraker KJ, Bidarra R (2010) Integrating procedural generation and manual editing of virtual worlds, pp 1-8

33. Russenschuck S (2010) Magnets for accelerators. In: Field Computation for Accelerator Magnets. Analytical and Numerical Methods for Electromagnetic Design and Optimization, Wiley-VCH, Berlin, pp 1-48

34. Mellert LD, Welte U (2012) Acoustical guidance in road tunnels. Presented at the 6th international conference tunnel safety and ventilation, pp187-194

35. Crawford A (1963) The perception of light signals: the effect of mixing flashing and steady irrelevant lights. Ergonomics 6(3):287-294

36. Boyce PR (1985) Movement under emergency lighting: the effect of illuminance. Light Res Technol 17(2):51-71

37. Wright M, Cook G, Webber G (2001) The effects of smoke on people's walking speeds using overhead lighting and wayguidance provision. In: Second international symposium on human behaviour in fire, Ulster

38. Haans A, Corbetta A, Kumar P, Toschi F (2017) Measuring the effect of dynamic lighting on pedestrian speed by means of overhead kinectTM sensors and continuous pedestrian tracking algorithms. In: 2017 International conference on environmental psychology (ICEP 2017), August 30-September 1, 2017, Coruña, Spain

39. Ronchi E, Nilsson D (2014) Traffic information signs, colour scheme of emergency exit portals and acoustic systems for road tunnel emergency evacuations. Department of Fire Safety Engineering, Lund University, Lund, Sweden, p. 3173

40. Galea ER, Xie H, Deere S, Cooney D, Filippidis L (2016) An international survey and full-scale evacuation trial demonstrating the effectiveness of the active dynamic signage system concept: evacuation trials examining active dynamic signage systems. Fire Mater 41:493-513

41. Vilar E, Rebelo F, Noriega P, Duarte Mayhorn CB (2014) Effects of competing environmental variables and signage on route-choices in simulated everyday and emergency wayfinding situations. Ergonomics 57(4):511-524

42. Xie H (2011) Investigation into the interaction of people with signage systems and its implementation within evacuation models. Ph.D. thesis, University of Greenwich, London, UK

43. Tahir N et al (2008) The CERN Super Proton Synchrotron as a tool to study high energy density physics. New J Phys 10(7):073028

44. Kinateder M, Müller M, Jost M, Mühlberger A, Pauli P (2014) Social influence in a virtual tunnel fire-influence of conflicting information on evacuation behavior. Appl Ergon 45(6):1649-1659 
45. Nilsson D, Frantzich H, Ronchi E, Fridolf K, Lindgren Walter A, Modig H (2018) Integrating evacuation research in large infrastructure tunnel projects - experiences from the Stockholm Bypass Project. Fire Saf J 97:119-125

46. Kinateder M, Warren WH (2016) Social influence on evacuation behavior in real and virtual environments. Front Robot AI 3:43

47. Moussaïd M et al (2016) Crowd behaviour during high-stress evacuations in an immersive virtual environment. J R Soc Interface 13(122):20160414

48. Tong D, Canter D (1985) The decision to evacuate: a study of the motivations which contribute to evacuation in the event of fire. Fire Saf J 9(3):257-265

49. Canter DV (1990) Fires and human behaviour. Fulton, London

50. Baldwin CL et al (2012) Multimodal cueing: the relative benefits of the auditory, visual, and tactile channels in complex environments. Proc Hum Factors Ergon Soc Annu Meet 56(1):1431-1435

51. Nilsson D, Boyce K (2015) Reviewing the ethical boundaries of empirical research in the area of human behaviour in fire. In: Human behaviour in fire symposium, proceedings, Cambridge, UK

52. Ribeiro J, Almeida JE, Rossetti RJF, Coelho AL (2012) Using serious games to train evacuation behaviour. In: 2012 7th Iberian conference presented at the proceedings of the information systems and technologies (CISTI), Madrid, Spain, 2012

Publisher's Note Springer Nature remains neutral with regard to jurisdictional claims in published maps and institutional affiliations. 